\title{
ON CENTRAL EXTENSIONS OF RATIONAL POINTS OF ALGEBRAIC GROUPS
}

\author{
BY VINAY V. DEODHAR \\ Communicated by Samuel Eilenberg, December 27, 1974
}

1. Introduction. The work announced here is an extension of the ideas of Steinberg [4] and Moore [2], where they consider split semisimple algebraic groups defined over a field $k$. One of the differences between such groups and the general semisimple groups (quasi-split groups in particular) is that the $k$-root system for the general groups is not necessarily reduced. A major aspect of the present work is the treatment of such cases. The details will be published elsewhere.

My sincere thanks are due to Professor M. S. Raghunathan for valuable guidance.

2. Notation. Let $k$ be an arbitrary field (with $|k| \geqslant 7$, but $\neq 9$ ). Let $G$ be a connected, simply connected, absolutely almost simple algebraic group defined over $k$. Let $G_{k}^{+}$be the group generated by the $k$-rational unipotent elements of $G$ which belong to the radical of a parabolic subgroup defined over $k$. Let $S \subset G$ be a maximal $k$-split torus and let $\mathfrak{g}=\mathfrak{h}_{0} \oplus_{\alpha \in \Phi} \mathfrak{g}_{\alpha}$ be the root space decomposition of the Lie algebra $g$ of $G$, with respect to $S$, where $\Phi$ is the $k$-root system. For $\alpha \in \Phi$, let $U^{(\alpha)}$ be the unipotent subgroup normalized by $S$ whose Lie algebra is $\oplus_{k>0} g_{k \alpha}$. Fix an order on $\Phi$ and let $U^{+}$(respectively $U^{-}$) be the unipotent subgroup normalized by $S$ corresponding to the positive roots (respectively negative roots) in this order.

3. Construction of the universal central extension (u.c.e.) of $G_{k}^{+}$. For the definition of u.c.e. and the (abstract) fundamental group (= Schur multiplier), see Steinberg [4]. We give an explicit construction of u.c.e. of $G_{k}^{+}$in terms of the groups $U_{k}^{+}$and $U_{k}^{-}$. We start with the following technical lemma.

The ChaIN LemMa. Let $\alpha \in \Phi$ and $x \in U_{k}^{(\alpha)}$ be a nontrivial element. Then there exist elements $x_{i} \in U_{k}^{(\alpha)}, y_{i} \in U_{k}^{(-\alpha)} \quad(i \in Z)$ such that (i) $x_{0}=x$, (ii) $x_{i} y_{i} x_{i+1}=y_{j} x_{j+1} y_{j+1} \forall i, j \in Z$; we denote this element by $w$; (iii) the element $w$ belongs to the normalizer $N(S)$ of $S$ and "acts" on $S$ as the reflec- 
tion with respect to $\alpha$; (iv) given any $x_{i}$ or $y_{j}$, the remaining elements of the chain $\left\{x_{m}, y_{n}\right\}$ are uniquely determined.

We now quotient the free product $U_{k}^{+} * U_{k}^{-}$by relations analogous to the ones in Steinberg [4] to get an extension $G^{\sim}$ of $G_{k}^{+}$. (We note that the chain lemma is essential for treating the rank 1 case.) We then have

THEOREM 1. $G^{\sim}$ is the universal central extension (u.c.e.) of $G_{k}^{+}$.

We denote the fundamental group by $\pi_{1}\left(G_{k}^{+}\right)$.

4. Computation of $\pi_{1}\left(G_{k}^{+}\right)$in case $G$ is quasi-split. Henceforth, we assume $G$ to be quasi-split over $k .\left(G_{k}^{+}=G_{k}\right.$ in this case.)

We define $M(k)$, which we call the Moore group of $k$, to be the fundamental group $\pi_{1}\left(\mathrm{SL}_{2}(k)\right)$ of $\mathrm{SL}_{2}(k)$. Moore [2] gives a presentation of this group in terms of pairs $(s, t) \in k^{*} \times k^{*}, k^{*}$ being $=k-\{0\}$. (It is then easily seen that $K_{2}$ of $k$ is related to $M(k)$.) Then we have

MAIN THEOREM. $\pi_{1}\left(G_{k}\right)$ is a quotient of $M(k)$.

In fact, we prove

THEOREM 2. There exists an injective homomorphism $i: \mathrm{SL}_{2}(k) \rightarrow G_{k}$ such that the induced homomorphism $i^{*}: \pi_{1}\left(\mathrm{SL}_{2}(k)\right) \rightarrow \pi_{1}\left(G_{k}\right)$ is surjective.

The important part of the proof of Theorem 2 is the handling of the cases in which $\Phi$ is not reduced. (For a complete list of such cases, see Tits [5, Table II].) It turns out that the existence of a root $\alpha \in \Phi$ such that $2 \alpha \in \Phi$ as well, is the cause of several difficulties which render the method used by Moore [2] inadequate and we have to use some new tools.

It is known (Steinberg [4]) that $M(k)$ for a finite field $k$ (with $|k| \geqslant 4$ ) is trivial. Thus as an immediate consequence to the main theorem, we have the following extension of a theorem of Steinberg [4].

THEOREM 3. If $k$ is finite (with $|k| \geqslant 7$ but $\neq 9$ ), then $\pi_{1}\left(G_{k}\right)$ is trivial, i.e. $G_{k}^{+}$is centrally closed.

5. Existence and computation of the (topological) fundamental group. Here we assume $k$ to be a local field. $G_{k}$ then acquires a topology with respect to which it is locally compact, 2 nd countable. One then has the notion of the (topological) u.c.e. $G_{k}^{c}$ and the fundamental group $\pi_{1}^{c}\left(G_{k}\right)$ of $G_{k}$ (cf. Moore [2]). Unlike the abstract case considered earlier, the existence of $G_{k}^{c}$ is not assured in this case. However, we have 
THEOREM 4. If $G$ is quasi-split over $k$, then $G_{k}^{c}$ exists. Moreover, $\left[G_{k}^{c}, G_{k}^{c}\right]=G_{k}^{c}$. The fundamental group $\pi_{1}^{c}\left(G_{k}\right)$ is cyclic. Further, if $k$ is nonarchimedean, then $\pi_{1}^{c}\left(G_{k}\right)$ is a quotient of $E_{k}$, the roots of unity in $k$ (and hence finite).

Moore [2] has considered an analogous problem in case of split groups. However, our methods to show the existence are different, and of necessity so. Once the existence is shown, the computation of $\pi_{1}^{c}\left(G_{k}\right)$ is an easy consequence of our main theorem and Moore's results.

6. The congruence subgroup problem for quasi-split groups. Here we assume $k$ to be a number field. We use the notation of Bass-Milnor-Serre [1]. We have the exact sequence:

$$
e \rightarrow C(S, G) \rightarrow \hat{G}_{k} \rightarrow \bar{G}_{k} \rightarrow e
$$

where $\hat{G}_{k}$ (respectively $\bar{G}_{k}$ ) is the completion of $G_{k}$ with respect to the $S$ arithmetic (respectively $S$-congruence) topology. We now have

THEOREM (RAghUNATHAN [3]). Let $G$ be a quasi-split of $k$-rank $\geqslant 2$ then the above extension (*) is central.

We now proceed along lines similar to Moore [2] and use the above theorem of Raghunathan along with results of $\$ 5$ to get

THEOREM 5. If $G$ is quasi-split of $k$-rank $\geqslant 2$, then $C(S, G)$ is a quotient of $E_{k}$, the roots of unity in $k$. Further, it is trivial if $S$ contains a nonarchimedean or a real place.

This theorem gives the solution of the congruence subgroup problem for quasi-split groups of $k$-rank $\geqslant 2$.

\section{REFERENCES}

1. H. Bass, J. Milnor and J.-P. Serre, Solution of the congruence subgroup problem for $\mathrm{SL}_{n}(n \geq 3)$ and $S p_{2 n}(n>2)$, Inst. Hautes Etudes Sci. Publ. Math. No. 33 (1967), 59-137. MR 39 \#5574.

2. C. C. Moore, Group extensions of p-adic and adelic linear groups, Inst. Hautes Etudes Sci. Publ. Math. No. 35 (1969), 157-222. MR 39 \#5575.

3. M. S. Raghunathan, On the congruence subgroup problem (to appear).

4. R. Steinberg, Générateurs, relations et revêtements de groupes algébriques, Colloq. Théorie des Groupes Algébriques (Bruxelles, 1962), Librairie Universitaire, Louvain; Gauthier-Villars, Paris, 1962, pp. 113-127. MR 27 \#3638.

5. J. Tits, Classification of algebraic semisimple groups, Proc. Sympos. Pure Math., vol. 9, Amer. Math. Soc., Providence, R. I., 1966, pp. 33-62. MR 37 \#309.

TATA INSTITUTE OF FUNDAMENTAL RESEARCH, COLABA, BOMBAY 400 005, INDIA 